\title{
Delayed and Misdiagnosed Complete Androgen Insensitivity Syndrome Cases in Two Sisters Which its Diagnose is Confirmed with Laparoscopic Gonadectomy
}

\author{
Keziban Doğan ${ }^{1}$, Hakan Güraslan ${ }^{1}$, Halil Fırat Baytekin ${ }^{2}$, Ali Yeşil ${ }^{1}$, Hediye Dağdeviren ${ }^{1}$ \\ ${ }^{1}$ Bakirköy Dr. Sadi Konuk Training and Research Hospital, Department of Obstetrics and Gynecology, Istanbul, Turkey. \\ ${ }^{2}$ Bakirköy Dr. Sadi Konuk Training and Research Hospital, Department of Pathology, Istanbul, Turkey
}

Received: 11 April 2015 accepted: 06 February 2016/ published online: 27 April 2016

(C) Ordu University Institute of Health Sciences, Turkey, 2016

\begin{abstract}
Complete androgen insensitivity syndrome (CAIS) is defined as end organ resistance to androgens with XY karyotype. Our aim is to present the diagnosis and treatment of a delayed and misdiagnosed CAIS case and to determine the importance of family history.

She was married woman with the complaint of infertility and primary amenorrhea. Patient who has undergone bilateral inguinal hernia operation; cannot be diagnosed with CAIS until the age of 25 . When the family history is investigated, it has been learned that two elder sisters menstruate but 17-year-old sister doesn't menstruate. It has been advised that she should apply to the university hospital, so she was diagnosed with CAIS for her.

Teenage girls' complaint of primary amenorrhea and bilateral inguinal hernia should be evaluated in terms of CAIS and family screening should be performed.
\end{abstract}

Key words: Complete androgen insensitivity syndrome, misdiagnose, inguinal hernia, family history.

\section{Address for correspondence/reprints: Keziban Doğan \\ E-mail address: drkzbn@yahoo.com}

Phone: +090 5356336207

DOI: $10.19127 /$ mbsjohs.98146

\section{Introduction}

Androgen insensitivity syndrome (AIS) characterized by a female phenotype with an XY karyotype which is a disorder of androgen resistance. The pathogenesis of AIS includes end organ insensitivity to androgens and defective androgen receptor gene locates on X-chromosome at Xq11-12 (Brinkmannet al., 2001). Gene mutations either inherits from the mother through the $\mathrm{X}$ chromosome recessively or occurs due to spontaneous mutations in the germ or egg cells (Hugeset al., 2006). It is a rarely seen syndrome, its incidence has been reported as $1 / 20.000$ to 1/64.000 (Grumbachet al., 2003). There are three major types of AIS; Complete androgen insensitivity syndrome (CAIS); known as also testicular feminization characterized by a female external genitalia, Partial androgen insensitivity 
syndrome (PAIS) characterized by a partially but not fully masculinized external genitalia that is called ambiguis genitalia and Minimal androgen insensitivity syndrome (MAIS) characterized by a normal male external genitalia that may be presented with infertility (Brinkmannet al., 2001). In CAIS cases, androgens are secreted from normal developing gonads, especially the testosterone level increases, the rising testosterone acts as substrate for estrogen synthesis, and the estrogen is responsible hormone for the feminization of prepubertal and postpubertal ages. The majority of CAIS patients admit to the hospital with the complaint of primary amenorrhea, inguinal mass containing testis and infertility (Sarpelet al., 2005).

Management of them should include multidisciplinary approach and gonadectomy to avoid gonadal tumors in later life, hormone replacement therapy (HRT), vaginal lengthening procedures in order to sustain the sex life if there is inadequate vaginal length and also psychological support in long-term basis (Cheikhelardet al., 2008). Family history should be considered as an important aspect to diagnose other cases of CAIS in the family.

\section{Case}

25-year -old married woman with complaint of infertility and primary amenorrhea admitted to our hospital which is a tertiary center. It has been learned that she admitted to a hospital with the complaint of amenorrhea when she was 12 years old. Oral contraceptive (OCD) was started but she didn't menstruate. In 2004, when she was 15 yearsold, she was operated from the left inguinal hernia under emergency conditions. She was admitted again to the hospital with complain of amenorrhea in 2008, she has been advised to use the OCD. She was operated again from right inguinal hernia in 2009. It has been reported that her uterus is much smaller than normal in magnetic resonance imaging (MRI) in 2013. She married two years ago and although unprotected sexual intercourse, she couldn't become pregnant. In addition, when questioning the patient it has been learned that she has 3 sisters, both of them are married, they menstruate and have children. The other sister was 17 years old and has no menstruation. In the physical examination; the patient's height was $170 \mathrm{~cm}$ and her weight was $66 \mathrm{~kg}$. The breast development was on the tanner stage III, there was no pubic and axillary hair. In the pelvic examination; labia minora, labia majora and the clitoris were in the normal development and appearance. It has seen that bilateral $3 \mathrm{~cm}$ scar tissues depend on the previous operation for bilateral inguinal hernia. Urethral orifice and the anus have normal appearance. In the vaginal examination; blind vagina $5-6 \mathrm{~cm}$ in length was determined, the cervix was not observed. Uterus and ovaries could not be assessed by vaginal ultrasound.

Her FSH was $2.68 \mathrm{mlU} / \mathrm{ml}$ (reference range for normal adult male: 1,27-19,2 $\mathrm{mlU} / \mathrm{ml})$, $\mathrm{LH}$ was $10,18 \mathrm{mlU} / \mathrm{ml}$ (reference range for normal adult male 1,24-8.62 $\mathrm{mlU} / \mathrm{ml}$ ), total serum testosterone was $3.17 \mathrm{ng} / \mathrm{dl}$ (reference range 1,75-7,81 $\mathrm{ng} / \mathrm{dl}$ for normal adult male, $0,1-0,75 \mathrm{ng} / \mathrm{dl}$ for normal adult female) and estradiol was $60,05 \mathrm{pg} / \mathrm{ml}$ (reference range for normal adult male: $<20-47 \mathrm{pg} / \mathrm{ml}$ ). 46 $\mathrm{XY}$ was detected in the karyotype analysis. The uterus and ovaries were not observed in the pelvic MRI and bilateral testes were detected in the inguinal canal entrance (Figure 1a, b, c). Because of all these findings, CAIS was diagnosed. She was informed for the risk of gonadal malignancy. The laparoscopic bilateral gonadectomy was planned.

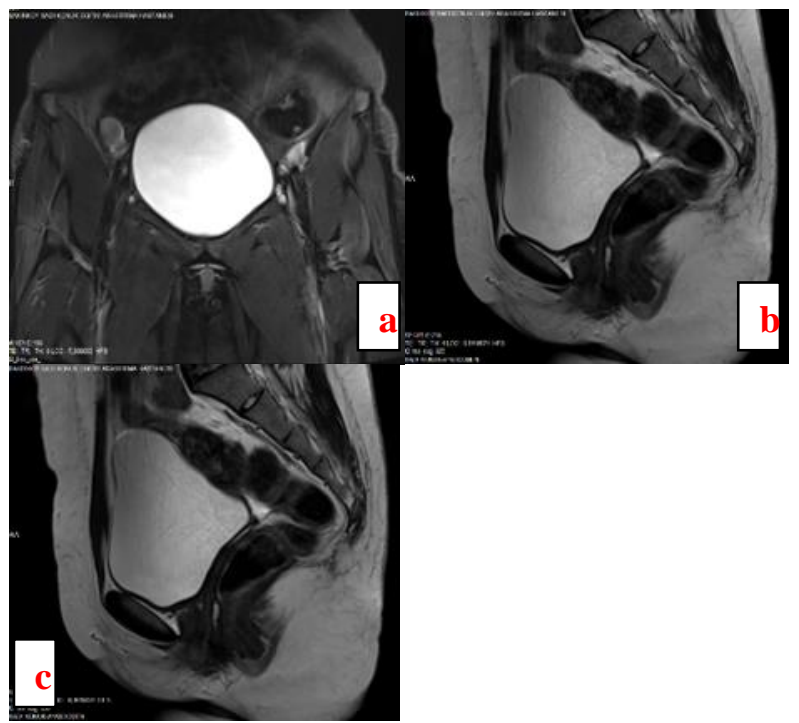

Figure 1 a, b, c: T2 weighed MRI image of left and right testis.

After standard preoperative preparation, diagnostic laparoscopy was performed under general anesthesia. Pelvic and abdominal inspection revealed no internal genitalia except bilateral gonads appearing as testis located in bilateral inguinal canals at the level of internal 
inguinal ring. The pedicles of the gonads were coagulated with bipolar diathermy and cut with laparoscopic scissors. Gonads were placed in endobags and removed intact after extending the port (Figure 2). No complications occurred during the operation. The patient was discharged on the following day after the surgery.

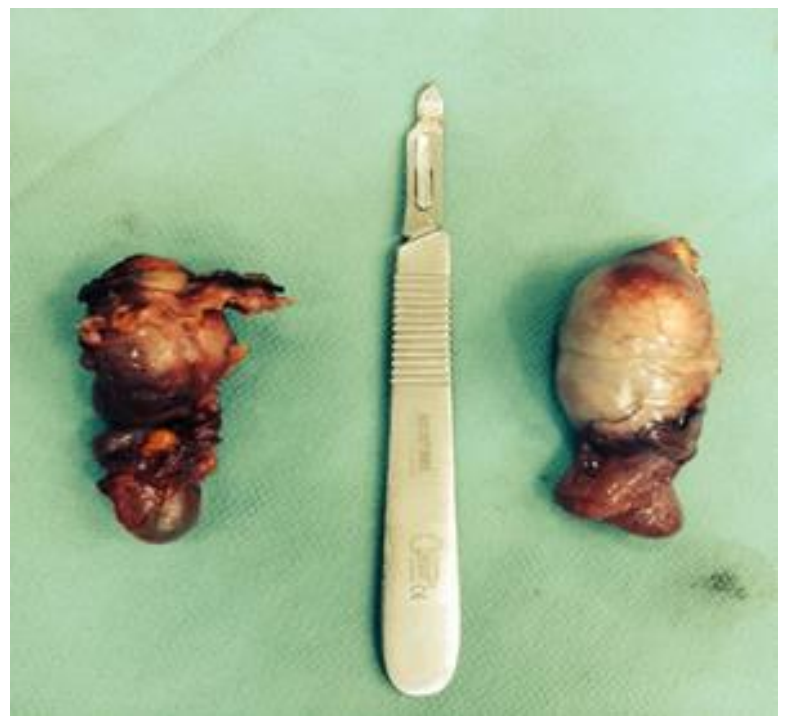

Figure 2: Photograph of excised gonads after gonadectomy

The histopathological examination of the gonads revealed thickened tunica albuginea, seminiferous tubules with primary and secondary spermatogonia and sertoli cells. Intertubular leydig cells were seen along with peritubular fibrosis (Figure 3).

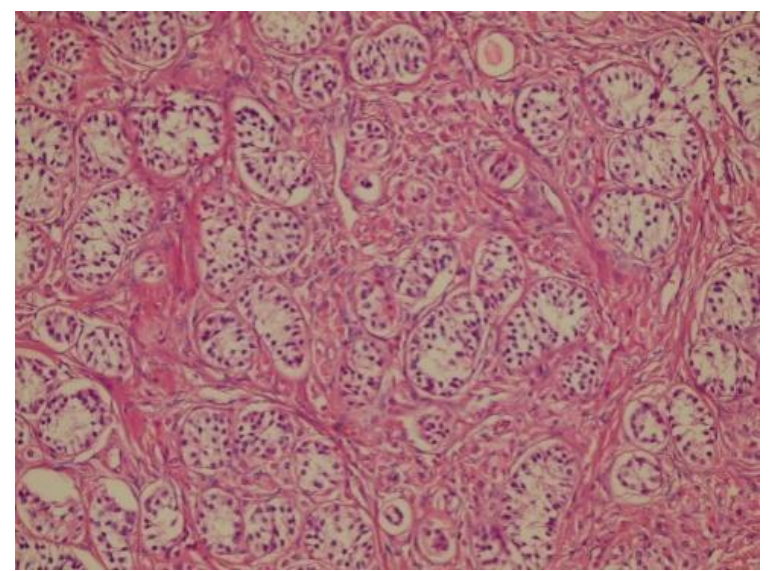

Figure3:

Microphotograph

showing

histopathology of testis.

After the clear diagnosis and operation of the patient, she has been told that her younger sister living in the rural area should be evaluated in terms of the CAIS. Her complains were the primary amenorrhea and bilateral inguinal swelling similar to our case. She was diagnosed with CAIS in an other university hospital.

\section{Discussion}

The diagnosis of CAIS is the first and most important problem as in our case. It is rarely seen syndrome, many tests including karyotyping are inadequate in order to differentiate from other genetic abnormalities, MRI is required in order to confirm the absence of uterus and ovaries and these tests are not frequently available in every hospital. The typical presentation mode of CAIS is an adolescent female who has breast development with pubertal growth but with no menarche or scanty growth of pubic and axillary hair. The breast development is a result of conversion of testosterone to estradiol. The other important clinical appearance of CAIS is bilateral inguinal or labial swellings in early childhood. The authors have found that $1-2 \%$ of the girls with inguinal hernias may have CAIS (Sarpelet al., 2005). Therefore, in a female child with bilateral inguinal hernia or labial swellings and also in pubertal and postpubertal ages with primer amenorrhea and external female seconder sex characters; the possibility of CAIS must always be kept in mind to prevent the delay on diagnosis or misdiagnosis. In CAIS cases, bilateral undecended testes carry a risk for malignancy especially germ cell tumors and the risk increases after puberty. Therefore; the standard treatment is gonadectomy to prevent possible malignant transformation of the testes during the late teenage years or early twenties that permits pubertal development spontaneously with the production of estrogen from the aromatization of the high levels of testosterone (Cheikhelardet al., 2008). Laparoscopic management that allow better visualization of abdomen and pelvis compared to laparotomy should be preferred due to minimal invasive surgery procedure with faster recovery times for the gonadectomy. Vaginal lengthening procedures can also be needed in some patients but we don't need these procedures because vaginal length is enough for sexual intercourse in our case. After gonadectomy; hormone replacement therapy is required for patient with CAIS to support the development and maintenance of secondary sexual characteristics and to prevent osteoporosis and psychological instability. Psychological and emotional support for the patient and their family should be available for a long-term basis from the time of diagnosis. Contact with other individuals who have androgen 
insensitivity syndrome is another important thing for psychological support (Jorgensenet al., 2010, Hiortet al., 2014).

The reason why the De novo mutations rate is more than $30 \%$ in patients with CAIS is the individuals with no family history (Leslie al., 1998). However, for the rest of the major patients group, detailed family history of CAIS case may provide early diagnosis and management of new cases in their family. Therefore a family screening of the affected individual should be performed.

\section{Conclusion}

In this case, a delayed and misdiagnosed of CAIS case was diagnosed on the basis of history of primary amenorrhea, infertility, and gynecologic examination and karyotyping. Ultrasonography and MRI imaging of the pelvis helped for the localization of gonads in inguinal canals. As the patient was in postpubertal age group, laparoscopic gonadectomy was carried out to prevent malignant transformation of gonads. HRT was continued and the patient and her husband were followed up by a multidisciplinary team of doctors for psychological and emotional support. Her sister with CAIS was diagnosed and treated by another medical center, because we informed the patient.

Informed Consent: Ethics committee approval was received for this study from Clinical Research Ethics Committee of Bakırköy Dr. Sadi Konuk Training and Research Hospital.

Peer-review: Externally peer-reviewed.

Author Contributions: Concept - KD, Design - KD, Supervision - HG, Materials - HRB, Data Collection and/or Processing - AY, HD; Analysis and/or Interpretation - KD; Literature Review - KD; Writing KD; Critical Review - KD.

Conflict of Interest: No conflict of interest was declared by the authors.

Financial Disclosure: The authors declared that this study hasn't received no financial support.

\section{References}

Brinkmann AO, Molecular basis of androgen insensitivity. Mol Cell Endocrinol. 2001; 20; 179:105-9.

Cheikhelard A, Morel Y, Thibaud E, Lortat-Jacob S, Jaubert F, Polak M et al. Long- term fallow up and comparison between genotype and phenotype in 29 cases of complete androgen insensitivity syndrome. J Urol 2008; 180; 4:1496-1501.

Grumbach MN, et al. Disorders of sex differentiation. In: Larsen PR, editor. Williams Textbook of Endocrinology. Philadelphia, Saunders, 2003, pp 842-1002

Hiort O, Birnbaum W, Marshall L, Wünsch L, Werner $\mathrm{R}$, Schröder $\mathrm{T}$ et al. Management of disorders of sex development. Nat Rev Endocrinol 2014; 10:520.

HugesIA, Deeb A. Androgen resistance. Best Pract Res Clin Endocrinol Metab 2006, 20:577-598

Jorgensen PB, Kjantansdottir KR, Fedder J. Care of women with XY karyotype: a clinical practice guideline. Fertil Steril 2010; 94:105.

Leslie ND. Haldane was right: de novo mutations in androgen insensitivity syndrome. J Pediatr 1998; 132:917-8.

Sarpel U, Palmer SK, Dolgin SE. The incidence of complete androgen insensitivity in girls with inguinal hernias and assessment of screening by vaginal length measurement. J Pediatr Surg 2005; 40:133 